1 Hacettepe Journal of Mathematics and Statistics

$\bigcap$ Volume 46 (3) (2017), 469-482

\title{
Approximation of fractional-order Chemostat model with nonstandard finite difference scheme
}

\author{
M. Zeinadini* and M. Namjoo ${ }^{\dagger \ddagger}$
}

\begin{abstract}
In this paper, the fractional-order form of three dimensional chemostat model with variable yields is introduced. The stability analysis of this fractional system is discussed in detail. In order to study the dynamic behaviours of mentioned fractional system, the well known nonstandard finite difference (NSFD) scheme is implemented. The proposed NSFD scheme is compared with the forward Euler and fourth order RungeKutta methods. Numerical results show that the NSFD approach is easy and accurate when applied to fractional-order chemostat model.
\end{abstract}

Keywords: Chemostat model, Fractional-order differential equation, Stability, Nonstandard finite difference scheme.

2000 AMS Classification: 37M05, 65L12, 65L20

Received : 23.09.2015 Accepted : 21.07.2016 Doi : 10.15672/HJMS.20174720337

\section{Introduction}

Competition modelling is one of the important topics in the mathematical biology. The simplest form of competition, however, occurs when individuals of different species compete for the same limited source foods, in some way species inhibit each other growth. This is called exploitative competition. A simple example of this type of competition occurs in a laboratory device, called a chemostat or a continuous culture, that models competition in a very simple lake [25].

As we know, most of the mathematical models of the biological systems are based on ordinary differential equations (ODEs) of integer-order. However, the behaviour of most biological systems has memory or after-effects, for which the ODEs of integer-order disregard such effects. It is worth noting that describing the behaviour of these systems by fractional-order differential equations is more useful than their classical integer-order

*Department of Mathematics, School of Mathematical Sciences, Vali-e-Asr University of Rafsanjan, Rafsanjan, Iran., Email: p91363001@post.vru.ac.ir

${ }^{\dagger}$ Department of Mathematics, School of Mathematical Sciences, Vali-e-Asr University of Rafsanjan, Rafsanjan, Iran., Email: namjoo@vru.ac.ir

$\ddagger_{\text {Corresponding Author. }}$ 
counterpart, due to good memory and hereditary properties of fractional derivatives. It should be emphasized that in the literature the fractional calculus has been used as an efficient tool to simulate the true nature of so many systems in diverse and widespread fields of science and engineering. For example, fractional calculus has been successfully applied to system biology $[2,3,6,8,9]$, physics [10, 11], chemistry and biochemistry [26], hydrology [14, 24], medicine [12, 5], and finance [7]. Hence study and use the fractional-order differential equations help us to have a better understanding of the biological systems behaviour. In the other hand, analytical solutions of these types of fractional equations cannot generally be obtained, hence good numerical schemes are playing important role in identifying the solution behaviour of such fractional equations and exploring their applications. Nevertheless, Among numericals methods, NSFD schemes can alternatively be used to obtain more qualitative results and remove numerical instabilities.

This paper organized as follows: In next Section, we elaborate the definition and some basic properties of Günwald-Letnikov (GL) approximation as well we discuss that how NSFD scheme can be implemented for systems of ODEs. In Section 3, fractional-order form of the chemostat model is introduced and also stability theorem and fractional Routh-Hurwitz stability conditions are given for the local asymptotic stability of the fractional-order systems. Section 4 is devoted to the study of the stability analysis of the fractional-order chemostat model. In Section 5, the idea of NSFD scheme for solving the fractional-order chemostat model is presented. Finally, the theoretical results obtained in former section are compared with the other numerical methods and the simulated numerical results are given.

\section{Preliminaries}

Although the discussion of the fractional calculus is as old as integer-order calculus, the complexity and the lack of applications postponed its progress till a few decades ago. Recently, most of the dynamical systems, based on the integer-order calculus, have been modified into the fractional-order domain due to the extra degrees of freedom and the flexibility which can be used to precisely fit the experimental data much better than the integer-order modelling.

2.1. Grünwald-Letnikov Approximation. Derivatives of fractional-order have been introduced in several ways. In this paper we consider GL approach. The GL method for the one-dimensional fractional derivative takes the following form [23]:

$$
\begin{aligned}
D^{\alpha} x(t) & =f(t, x(t)), \quad x(0)=x_{0}, \quad t \in\left[0, t_{f}\right], \\
D^{\alpha} x(t) & =\lim _{h \rightarrow 0} h^{-\alpha} \sum_{j=0}^{\left[\frac{t}{h}\right]}(-1)^{j}\left(\begin{array}{c}
\alpha \\
j
\end{array}\right) x(t-j h),
\end{aligned}
$$

where $D^{\alpha}, h$ and $[t / h]$ denote fractional derivative, stepsize and the integer part of $t / h$, respectively. Therefore, the Eq. (2.1) is discretized as follows:

$$
\sum_{j=0}^{n} c_{j}^{\alpha} x_{n-j}=f\left(t_{n}, x_{n}\right), \quad n=1,2,3, \ldots
$$

where $t_{n}=n h, x_{n-j}$ is approximation of $x\left(t_{n-j}\right)$ and $c_{j}^{\alpha}, \quad j=1,2 \ldots, n$ are the binomial coefficients that are defined as:

$$
c_{j}^{\alpha}=\left(1-\frac{1+\alpha}{j}\right) c_{j-1}^{\alpha}, \quad c_{0}^{\alpha}=h^{-\alpha}, \quad j=1,2,3, \ldots, n .
$$


2.2. Nonstandard Finite Difference Schemes. NSFD schemes were firstly proposed by Mickens[20, 21] for ODEs and successively, their use has been investigated in several fields. To describe NSFD scheme, we consider an ODE such as

$$
\frac{d x}{d t}=f(t, x, \lambda), \quad x(0)=x_{0}, \quad t \in\left[0, t_{f}\right],
$$

where $\lambda$ is a parameter. Given a discretization $t_{n}=n h$, NSFD is constructed by following two main steps:

(i) The derivative at the left-hand side of the Eq. (2.2) is replaced by a discrete form

$$
\frac{d x}{d t} \approx \frac{x_{n+1}-x_{n}}{\phi(h, \lambda)},
$$

where $x_{n}$ is an approximation of $x\left(t_{n}\right)$.

(ii) The nonlinear term in the Eq. (2.2) is replaced by a nonlocal discrete representation $F\left(t, x_{n+1}, x_{n}, \ldots, \lambda\right)$ depending on some of the previous approximation. Hence the gained scheme described as follows:

$$
\frac{x_{n+1}-x_{n}}{\phi(h, \lambda)}=F\left(t, x_{n+1}, x_{n}, \ldots, \lambda\right) .
$$

The discrete derivative on the left-hand side of the Eq.(2.4) is a generalization of the classical discrete representation for the first derivative that is obtained by using $\phi(h, \lambda)=$ $h$. The denominator function $\phi(h, \lambda)$, that is function of stepsize, must have consistency condition

$$
\phi(h, \lambda)=h+\mathrm{O}\left(h^{2}\right),
$$

to ensure that the discrete representation of (2.3) converges to the corresponding continuous derivative as $h \rightarrow 0$. Examples of denominator function that satisfy the condition (2.5) are $h, \sin (h), 1-e^{-h},\left(1-e^{-\lambda h}\right) / \lambda$ and so forth. The papers of Mickens [16, 17, 19] give a general procedure for determining $\phi(h)$ for systems of ODEs. In general, for an ODE with polynomial terms,

$$
\frac{d x}{d t}=a x+(N L), \quad \mathrm{NL} \equiv \text { Nonlinearterms }
$$

the NSFD discretization for the linear expression is given by Mickens [16]

$$
\frac{x_{n+1}-x_{n}}{\phi}=a x_{n}+(N L)_{n},
$$

where the denominator function is

$$
\phi(h, a)=\frac{e^{a h}-1}{a} .
$$

Note that if $a=0$ then the denominator function is just $h$, i.e., $\phi(h)=h$.

The first NSFD requirement is that the dependent functions should be modelled nonlocally on the discrete-time computational grid. Particular examples of this include the following functions $[17,18]$

$$
\begin{aligned}
& x y \approx x_{n+1} y_{n} \quad x y \approx x_{n} y_{n+1}, \\
& x^{2} \approx 2 x_{n+1} x_{n}, \quad x^{2} \approx 2 x_{n} \frac{x_{n+1}+x_{n}}{2} .
\end{aligned}
$$


By applying this technique and using the GL discretization method, it yields the following relation

$$
x_{n+1}=\frac{-\sum_{j=1}^{n+1} c_{j}^{\alpha} x_{n+1-j}+f\left(t_{n+1}, x_{n+1}\right)}{c_{0}^{\alpha}}, \quad n=0,1,2, \ldots
$$

where $c_{0}^{\alpha}=\phi(h)^{-\alpha}$.

\section{Fractional-Order Chemostat Model}

At time $t$, let $s(t)$ denotes the concentration of nutrient in the vessel, $x(t)$ and $y(t)$ denote the concentration of two microorganism. The mathematical model takes the form

$$
\begin{aligned}
& \frac{d s}{d t}=\left(s_{0}-s\right) Q-\frac{1}{\delta_{1}}\left(\frac{m_{1} s}{k_{1}+s}-L\right) x-\frac{1}{\delta_{2}} \frac{m_{2} s}{k_{2}+s} y, \\
& \frac{d x}{d t}=x\left(\frac{m_{1} s}{k_{1}+s}-L-Q\right), \\
& \frac{d y}{d t}=y\left(\frac{m_{2} s}{k_{2}+s}-Q\right), \\
& s(0)=s_{0}, \quad x(0)=x_{0}, \quad y(0)=\mathrm{y}_{0},
\end{aligned}
$$

where $s_{0}$ is the input concentration of nutrient, $Q$ is the washout rate, $m_{i}$ is the maximal growth rates, $k_{i}$ is the Michaelis-Menton constants, $\delta_{i}$ is the yield coefficients and $L$ is the intrinsic consumption rate for the first microorganism, which are all positive. This model is usually called the Monod model or the model with Michaelis Menten dynamics $[4,13,22]$.

Here we investigate system Eqs. (3.1) with yield coefficients $\delta_{1}=A+B s, \delta_{2}=C+D s^{3}$, which means that the production of the microbial biomasses is very sensitive to the concentration of the nutrient. In the system Eqs. (3.1) we have used the growth rate functions

$$
F_{i}(s)=\frac{m_{i} s}{k_{i}+s}, \quad i=1,2,
$$

which have following common features:

(i) $F_{i}(0)=0$.

(ii) $F_{i}$ is an increasing function of $s$.

(iii) $F_{i}$ approaches to a constant value as $s$ approaches to infinity.

Now we introduce fractional-order form of the system Eqs. (3.1). The new system is described by the following set of fractional differential equations of order $\alpha_{1}, \alpha_{2}, \alpha_{3}>0$, with initial population; i.e., $s(0)>0, \quad x(0)>0, \quad y(0)>0$.

$$
\begin{aligned}
& D^{\alpha_{1}} s(t)=\left(s_{0}-s\right) Q-\frac{1}{\delta_{1}}\left(\frac{m_{1} s}{k_{1}+s}-L\right) x-\frac{1}{\delta_{2}} \frac{m_{2} s}{k_{2}+s} y, \\
& D^{\alpha_{2}} x(t)=x\left(\frac{m_{1} s}{k_{1}+s}-L-Q\right), \\
& D^{\alpha_{3}} y(t)=y\left(\frac{m_{2} s}{k_{2}+s}-Q\right), \\
& s(0)=s_{0}, \quad x(0)=x_{0}, \quad y(0)=y_{0}, \\
& 0<\alpha_{i} \leq 1, \quad i=1,2,3 .
\end{aligned}
$$


In order to analyze the stability of the model, stability theorem on fractional-order systems and fractional Routh-Hurwitz stability conditions are introduced.

3.1. Theorem. [15] Consider the following commensurate fractional-order system:

$$
D^{\alpha} x(t)=f(x(t)), \quad x(0)=x_{0}, \quad t \in\left[0, t_{f}\right],
$$

where, $0<\alpha \leq 1$ and $x \in \mathbb{R}^{n}$. Equilibrium point $E$ of the system (3.3), calculated by solving $f(x)=0$, is locally asymptotically stable if all eigenvalues of the Jacobian matrix $J \equiv \frac{\partial f}{\partial x}$ that evaluated at the equilibrium point E, satisfy:

$$
|\arg (\lambda)|>\alpha \frac{\pi}{2}
$$

3.2. Proposition. [1] Suppose $P(\lambda)=\lambda^{2}+b \lambda+c$ is characteristic polynomial of the Jacobian matrix $\frac{\partial f}{\partial x}$, evaluated at the equilibrium point E. For $0<\alpha \leq 1$, the eigenvalues of Jacobian matrix $J \equiv \frac{\partial f}{\partial x}$, satisfy condition (3.4) in Theorem 3.1 if

$$
b>0, \quad c>0,
$$

or

$$
b \leq 0, \quad 4 c>b^{2}, \quad\left|\tan ^{-1}\left(\sqrt{4 c-b^{2}} / b\right)\right|>\alpha \frac{\pi}{2} .
$$

\section{Stability Analysis of the Equilibrium Points}

In this section we deal with stability of the equilibrium points of the system Eqs. (3.2). The equilibrium points of this system are:

$$
\begin{aligned}
& E_{0}=\left(s_{0}, 0,0\right), \\
& E_{1}=\left(\beta_{1},\left(s_{0}-\beta_{1}\right)\left(A+B \beta_{1}\right), 0\right), \\
& E_{2}=\left(\beta_{2}, 0,\left(s_{0}-\beta_{2}\right)\left(C+D \beta_{2}^{3}\right)\right),
\end{aligned}
$$

where

$$
\beta_{1}=\frac{k_{1}(L+Q)}{m_{1}-(L+Q)}, \quad \beta_{2}=\frac{Q k_{2}}{m_{2}-Q} .
$$

Note that the equilibrium points $E_{i}, \quad i=0,1,2$, have real biological meaning if their components are non-negative. Since $s_{0}>0$, the equilibrium point $E_{0}$ exists by biological meaning. Also the equilibrium points $E_{2}$ and $E_{3}$ have real biological meaning when $0<\beta_{i}<s_{0}, \quad i=1,2$. Now let us verify stability of these equilibrium points. The Jacobian matrix of the system (3.2) at the equilibrium point $E=(s, x, y)$ is

$$
J(s, x, y)=\left[\begin{array}{ccc}
T(s, x, y) & -\frac{1}{A+B s}\left(\frac{m_{1} s}{k_{1}+s}-L\right) & -\frac{1}{C+D s^{3}} \frac{m_{2} s}{k_{2}+s} \\
\frac{m_{1} k_{1} x}{\left(k_{1}+s\right)^{2}} & \frac{m_{1} s}{k_{1}+s}-L-Q & 0 \\
\frac{m_{2} k_{2} y}{\left(k_{2}+s\right)^{2}} & 0 & \frac{m_{2} s}{k_{2}+s}-Q
\end{array}\right],
$$

where

$$
\begin{aligned}
T(s, x, y)= & -Q-\frac{x}{A+B s} \frac{m_{1} k_{1}}{\left(k_{1}+s\right)^{2}}+\frac{B x}{(A+B s)^{2}}\left(\frac{m_{1} s}{k_{1}+s}-L\right) \\
& -\frac{y}{C+D s^{3}} \frac{k_{2} m_{2} y}{k_{2}+s}+\frac{3 D s^{2} y}{\left(C+D s^{3}\right)^{2}} \frac{m_{2} s}{k_{2}+s} .
\end{aligned}
$$

The characteristic equation of the Jacobian matrix $J$ at the equilibrium point $E_{0}$ is

$$
P(\lambda)=(\lambda+Q)\left(\lambda^{2}+b_{1} \lambda+c_{1}\right)=0,
$$

where

$$
b_{1}=L+2 Q-\left(\frac{m_{1} s_{0}}{k_{1}+s_{0}}+\frac{m_{2} s_{0}}{k_{2}+s_{0}}\right), \quad c_{1}=\left(L+Q-\frac{m_{1} s_{0}}{k_{1}+s_{0}}\right)\left(Q-\frac{m_{2} s_{0}}{k_{2}+s_{0}}\right) .
$$


Eigenvalues of the matrix $J$ at the equilibrium point $E_{0}$ are

$$
\lambda_{1}=-Q, \quad \lambda_{2}=-\left(L+Q-\frac{m_{1} s_{0}}{k_{1}+s_{0}}\right), \quad \lambda_{3}=-\left(Q-\frac{m_{2} s_{0}}{k_{2}+s_{0}}\right) .
$$

These eigenvalues are real, hence by Theorem 3.1 the equilibrium point $E_{0}$ is stable if $\lambda_{i}<0, \quad i=1,2,3$. Since $Q>0$, the inequality $\lambda_{1}<0$ holds. Also inequalities $\lambda_{2}<0$ and $\lambda_{3}<0$ are satisfied when $\beta_{i}>s_{0}, \quad i=1,2$. Therefore the equilibrium point $E_{0}$ is stable if

$$
\beta_{i}>s_{0}, \quad i=1,2 .
$$

The characteristic equation of the Jacobian matrix $J$, evaluated at the equilibrium point $E_{1}$, is

$$
P(\lambda)=\left(\lambda-a_{2}\right)\left(\lambda^{2}+b_{2} \lambda+c_{2}\right)=0
$$

where

$$
\begin{aligned}
& a_{2}=\frac{m_{2} \beta_{1}}{k_{2}+\beta_{1}}-Q, \\
& b_{2}=Q-\left(s_{0}-\beta_{1}\right)\left(\frac{B Q}{A+B \beta_{1}}-\frac{m_{1} k_{1}}{\left(k_{1}+\beta_{1}\right)^{2}}\right), \\
& c_{2}=\left(s_{0}-\beta_{1}\right) \frac{m_{1} k_{1}}{\left(k_{1}+\beta_{1}\right)^{2}} Q .
\end{aligned}
$$

To consider stability of the equilibrium point $E_{1}$, Let

$$
R_{1}=\frac{Q\left(s_{0}-\beta_{1}\right)\left(k_{1}+\beta_{1}\right)^{2}-Q\left(k_{1}+\beta_{1}\right)^{2}-m_{1} k_{1} \beta_{1}\left(s_{0}-\beta_{1}\right)}{Q\left(k_{1}+\beta_{1}\right)^{2}+m_{1} k_{1}\left(s_{0}-\beta_{1}\right)} .
$$

Note that $a_{2}$ is a real root of the characteristic equation of the Jacobian matrix $J$ at the equilibrium point $E_{1}$, therefore conditions $a_{2}<0$, or equivalently $\beta_{1}<\beta_{2}$ and $c_{2}>0$, are necessary for stability of the equilibrium point $E_{1}$ ( Theorem 3.1 and Proposition 3.2). Since all parameters in chemostat model are positive and $0<\beta_{1}<s_{0}$, the condition $c_{2}>0$ holds. We now consider the following two cases:

(i) If $b_{2}>0$ or equivalently $\frac{A}{B}>R_{1}$ then by Theorem $3.1, E_{1}$ is stable equilibrium point of the system Eqs. (3.2).

(ii) If $b_{2} \leq 0$ or equivalently $\frac{A}{B} \leq R_{1}$ and

$$
4 c_{2}>b_{2}^{2}, \quad\left|\tan ^{-1}\left(\frac{\sqrt{4 c_{2}-b_{2}^{2}}}{b_{2}}\right)\right|>\alpha \frac{\pi}{2},
$$

then the equilibrium point $E_{1}$ is stable ( Proposition 3.2).

In case (ii) the second condition in (4.2), that satisfy the first condition, is equivalent to

$$
4 \cos ^{2}\left(\alpha \frac{\pi}{2}\right) c_{2}>b_{2}^{2}
$$

If we let

$$
R_{2}=\frac{Q\left(s_{0}-\beta_{1}\right)\left(k_{1}+\beta_{1}\right)^{2}}{\left(Q+2 \cos \left(\alpha \frac{\pi}{2}\right) \sqrt{c_{2}}\right)\left(k_{1}+\beta_{1}\right)^{2}+m_{1} k_{1}\left(s_{0}-\beta_{1}\right)}-\beta_{1},
$$

then case (ii) follows that $R_{2}<\frac{A}{B} \leq R_{1}$.

The characteristic equation of the equilibrium point $E_{2}$ is

$$
P(\lambda)=\left(\lambda-a_{3}\right)\left(\lambda^{2}+b_{3} \lambda+c_{3}\right)=0,
$$

where

$$
\begin{aligned}
a_{3} & =\frac{m_{1} \beta_{2}}{k_{1}+\beta_{2}}-L-Q, \\
b_{3} & =Q-\left(s_{0}-\beta_{2}\right)\left(\frac{3 D \beta_{2}{ }^{2}}{C+D \beta_{2}^{3}}-\frac{m_{2} k_{2}}{\left(k_{2}+\beta_{2}\right)^{2}}\right), \\
c_{3} & =\left(s_{0}-\beta_{2}\right) \frac{m_{2} k_{2}}{\left(k_{2}+\beta_{2}\right)^{2}} Q .
\end{aligned}
$$


Let

$$
R_{3}=\frac{3 \beta_{2}^{2}\left(s_{0}-\beta_{2}\right)\left(k_{2}+\beta_{2}\right)^{2}-\beta_{2}^{3} Q\left(k_{2}+\beta_{2}\right)^{2}-\beta_{2}^{3}\left(s_{0}-\beta_{2}\right) m_{2} k_{2}}{Q\left(k_{2}+\beta_{2}\right)^{2}+\left(s_{0}-\beta_{2}\right) m_{2} k_{2}},
$$

like equilibrium point $E_{1}$, conditions $a_{3}<0$ and $c_{3}>0$ are necessary for stability of the equilibrium point $E_{2}$. Positivity of the parameters and condition $\beta<s_{0}$, show that $c_{3}>0$. But inequality $a_{3}<0$ holds provided that $\beta_{2}<\beta_{1}$. Now, we have the following two cases:

(i) If $b_{3}>0$ ( equivalently $\frac{C}{D}>R_{3}$ ) then by Theorem 3.1 the equilibrium point $E_{2}$ is stable.

(ii) If $\left.-2 \sqrt{c_{3}} \cos \left(\alpha \frac{\pi}{2}\right)\right)<b_{3} \leq 0$ then the equilibrium point $E_{2}$ is stable (Proposition 3.2 ).

Like equilibrium point $E_{1}$, case (ii) is equal to $R_{4}<\frac{C}{D} \leq R_{3}$, where

$$
R_{4}=\frac{3 \beta_{2}^{2}\left(s_{0}-\beta_{2}\right)\left(k_{2}+\beta_{2}\right)^{2}}{\left(Q+2 \cos \left(\alpha \frac{\pi}{2}\right) \sqrt{c_{3}}\right)\left(k_{2}+\beta_{2}\right)^{2}+m_{2} k_{2}\left(s_{0}-\beta_{2}\right)}-\beta_{2}^{3} .
$$

4.1. Theorem. Let $0<\alpha \leq 1$, then for the equilibrium points $E_{0}, E_{1}$ and $E_{2}$ of system Eqs. (3.2), the following statements hold.

(i) If $\beta_{i}>s_{0}, \quad i=1,2$, then the equilibrium point $E_{0}$ is stable.

(ii) If $\beta_{1}<\beta_{2}$ and $\frac{A}{B}>R_{1}$ or $\beta_{1}<\beta_{2}$ and $R_{2}<\frac{A}{B} \leq R_{1}$, then the equilibrium point $E_{1}$ is stable.

(iii) If $\beta_{2}<\beta_{1}$ and $\frac{C}{D}>R_{3}$ or $\beta_{2}<\beta_{1}$ and $R_{4}<\frac{C}{D} \leq R_{3}$, then the equilibrium point $E_{2}$ is stable.

\section{NSFD Scheme for Fractional-Order Chemostat Model}

In this section, we present numerical simulation to illustrate the results obtained in the previous section. By using definition of GL derivative and use NSFD scheme for the model we have:

$$
\begin{aligned}
& \sum_{j=0}^{n+1} c_{j}^{\alpha_{1}} s_{n+1-j}=\left(s_{0}-s_{n+1}\right) Q-\frac{1}{A+B s_{n}}\left(\frac{m_{1} s_{n+1}}{k_{1}+s_{n}}-L\right) x_{n} \\
& -\frac{1}{C+D\left(s_{n}\right)^{3}} \frac{m_{2} s_{n+1} y_{n}}{k_{2}+s_{n}}, \\
& \sum_{j=0}^{n+1} c_{j}^{\alpha_{2}} x_{n+1-j}=x_{n}\left(\frac{m_{1} s_{n+1}}{k_{1}+s_{n+1}}\right)-(L+Q) x_{n+1}, \\
& \sum_{j=0}^{n+1} c_{j}^{\alpha_{3}} y_{n+1-j}=y_{n} \frac{m_{2} s_{n+1}}{k_{2}+s_{n+1}}-Q y_{n+1},
\end{aligned}
$$

where $t_{n}=n h$ and $c_{j}^{\alpha_{i}}, \quad i=1,2,3$ are the GL coefficients defined as:

$$
c_{j}^{\alpha_{i}}=\left(1-\frac{1+\alpha_{i}}{j}\right) c_{j-1}^{\alpha_{i}}, \quad c_{0}^{\alpha_{i}}=\left(\phi_{i}(\mathrm{~h})\right)^{-\alpha_{i}}, \quad j=1,2,3, \ldots, n+1, \quad i=1,2,3,
$$

with

$$
\phi_{1}(h)=h, \quad \phi_{2}(h)=h, \quad \phi_{3}(h)=h .
$$

The previous discretization of system Eqs. (3.2) has the following principles:

(i) $\phi_{i}$, is an increasing function of $h$ for $i=1,2,3$.

(ii) Comparing to the system Eqs. (3.2) in the first equation $-s$ changed to $-s_{n+1}$ and in the second and the third equations changed to $s_{n+1}$. 
(iii) In the second equation $-x$ changed to $x_{n+1}$ and in the third equation $-y$ changed to $y_{n+1}$.

Manipulating previous discretization we get the following equations:

$$
\begin{aligned}
s_{n+1}= & \frac{-\sum_{j=1}^{n+1} c_{j}^{\alpha_{i}} s_{n+1-j}+s_{0} Q+\frac{L x_{n}}{A+B s_{n}}}{c_{0}^{\alpha_{i}}+Q+\frac{m_{1} x_{n}}{\left(A+B s_{n}\right)\left(k_{1}+s_{n}\right)}+\frac{m_{2} y_{n}}{\left(C+D s_{n}^{3}\right)\left(k_{2}+s_{n}\right)}}, \\
x_{n+1}= & \frac{-\sum_{j=1}^{n+1} c_{j}^{\alpha_{i}} x_{n+1-j}+x_{n}\left(\frac{m_{1} s_{n+1}}{k_{1}+s_{n}}\right)}{c_{0}^{\alpha_{i}}+L+Q} \\
y_{n+1}= & \frac{-\sum_{j=1}^{n+1} c_{j}^{\alpha_{i}} y_{n+1-j}+y_{n} \frac{m_{2} s_{n+1}}{k_{2}+s_{n+1}}}{c_{0}^{\alpha_{i}}+Q}
\end{aligned}
$$

5.1. Proposition. The numerical solutions obtained from system (5.1) for case $0<$ $\alpha_{i} \leq 1, \quad i=1,2,3$ satisfy

$$
\begin{aligned}
& s_{n}>0 \\
& x_{n}>0 \\
& y_{n}>0
\end{aligned} \Rightarrow \begin{aligned}
& s_{n+1}>0 \\
& x_{n+1}>0 \\
& y_{n+1}>0
\end{aligned}
$$

Proof. Since $c_{0}^{\alpha_{i}}>0$, by recursive relation

$$
c_{j}^{\alpha_{i}}=\left(1-\frac{1+\alpha_{i}}{j}\right) c_{j-1}^{\alpha_{i}}, \quad j=1,2, \ldots, n+1,
$$

we have $c_{j}^{\alpha_{i}}<0, \quad j>0$, therefore the system equations (5.1) show that relation (5.2) is established. For case $\alpha_{i}=1, i=1,2,3$, by considering the following system

$$
\begin{aligned}
\frac{s_{n+1}-s_{n}}{\phi_{1}(h)}= & \left(s_{0}-s_{n+1}\right) Q-\frac{1}{A+B s_{n}}\left(\frac{m_{1} s_{n+1}}{k_{1}+s_{n}}-L\right) x_{n} \\
& -\frac{1}{C+D\left(s_{n}\right)^{3}} \frac{m_{2} s_{n+1} y_{n}}{k_{2}+s_{n}} \\
\frac{x_{n+1}-x_{n}}{\phi_{2}(h)} & =x_{n}\left(\frac{m_{1} s_{n+1}}{k_{1}+s_{n+1}}\right)-(L+Q) x_{n+1}, \\
\frac{y_{n+1}-y_{n}}{\phi_{3}(h)} & =y_{n} \frac{m_{2} s_{n+1}}{k_{2}+s_{n+1}}-Q y_{n+1} .
\end{aligned}
$$

and solving this system in terms of $s_{n+1}, x_{n+1}, y_{n+1}$ we conclude that relation (5.2) holds.

\section{Numerical results}

Analytical studies always remain incomplete without numerical verification of the results. In this section we present numerical simulation to illustrate the result obtained in previous sections. Now we consider the fractional-order chemostat model in several cases. For the parameter values $m_{1}=1.625, m_{2}=1.875, k_{1}=1, k_{2}=1.25, Q=1$ and initial conditions $s_{0}=1, x_{0}=0.5, y_{0}=0.4$, numerical solutions of the system Eqs. (3.2) converge to the equilibrium point $E_{0}$ (Fig. 1). These values satisfy conditions $\beta_{1}>s_{0}$ and $\beta_{2}>s_{0}$ (for these set of data $\beta_{1}=1.6860$ and $\beta_{2}=1.4286$ ). For parameter values $A=0.0005, B=1.3, k_{1}=0.53, k_{2}=1, m_{1}=1.05, L=0.002, Q=0.5$ and initial conditions $s_{0}=1.4, x_{0}=0.1, y_{0}=0.4$, numerical solutions of the system Eqs.(3.2) converge to the equilibrium point $E_{1}$ (Fig. 2). For these values we have $\frac{A}{B}>R_{1}$ and $\beta_{1}<\beta_{2}$ that ensure solutions converge to the equilibrium point $E_{1}$ (for this set of data $\beta_{1}=0.9161, \beta_{2}=1.25, \frac{A}{B}>0$ and $R_{1}<0$ ). Finally for parameter values $C=2$, 

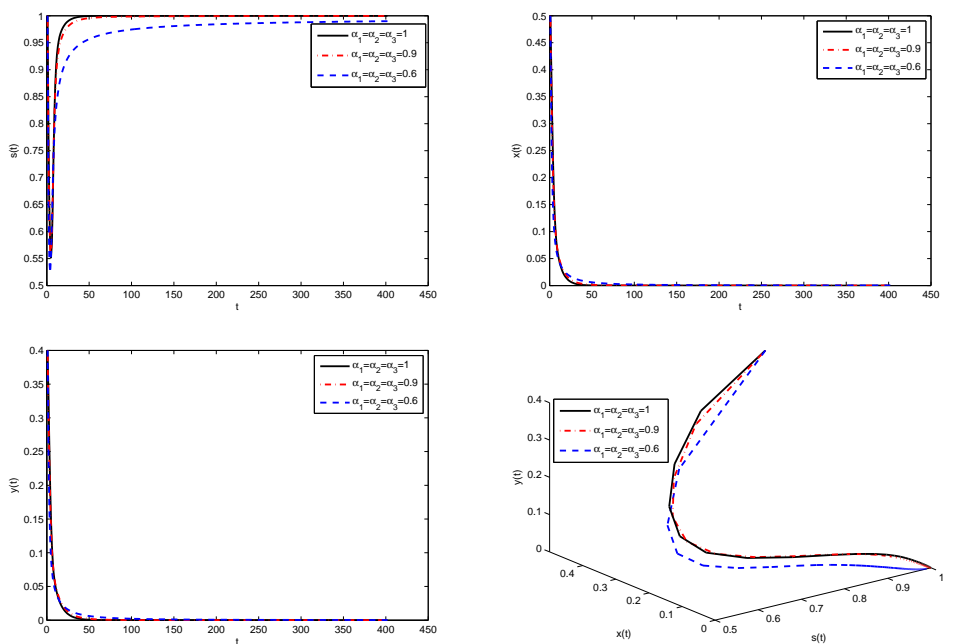

Figure 1. Numerical solutions of the system Eqs. (3.2) converge to the equilibrium point $E_{0}$ for different $\alpha_{i}$ with stepsize $h=1.5$.
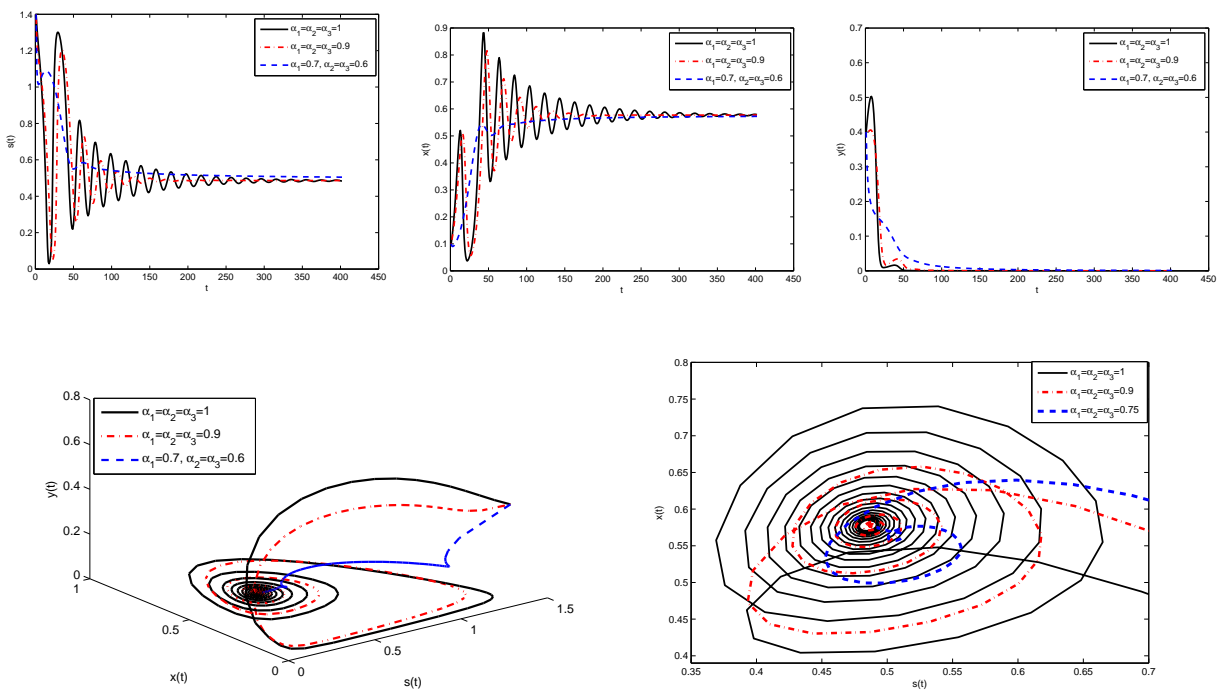

Figure 2. Numerical solutions of the system Eqs. (3.2) converge to the equilibrium point $E_{1}$ for different $\alpha_{i}$ with stepsize $h=1.5$.

$D=1.5, k_{2}=0.75, m_{2}=1.5, L=0.001, Q=1$ and initial conditions $s_{0}=2.2, x_{0}=0.5$, $y_{0}=0.4$, we have $\frac{C}{D}>R_{2}$ and $\beta_{1}>\beta_{2}$ (for this set of data, $\frac{C}{D}=1.3333, R_{2}=-.649$, $\beta_{1}=4.0201$ and $\left.\beta_{2}=1.5\right)$. With these conditions numerical solutions converge to the equilibrium point $E_{2}$. All numerical solution illustrated in Figs. 1 3, gained by step size $h=1.5$, this step size shows high accuracy of NSFD scheme. 

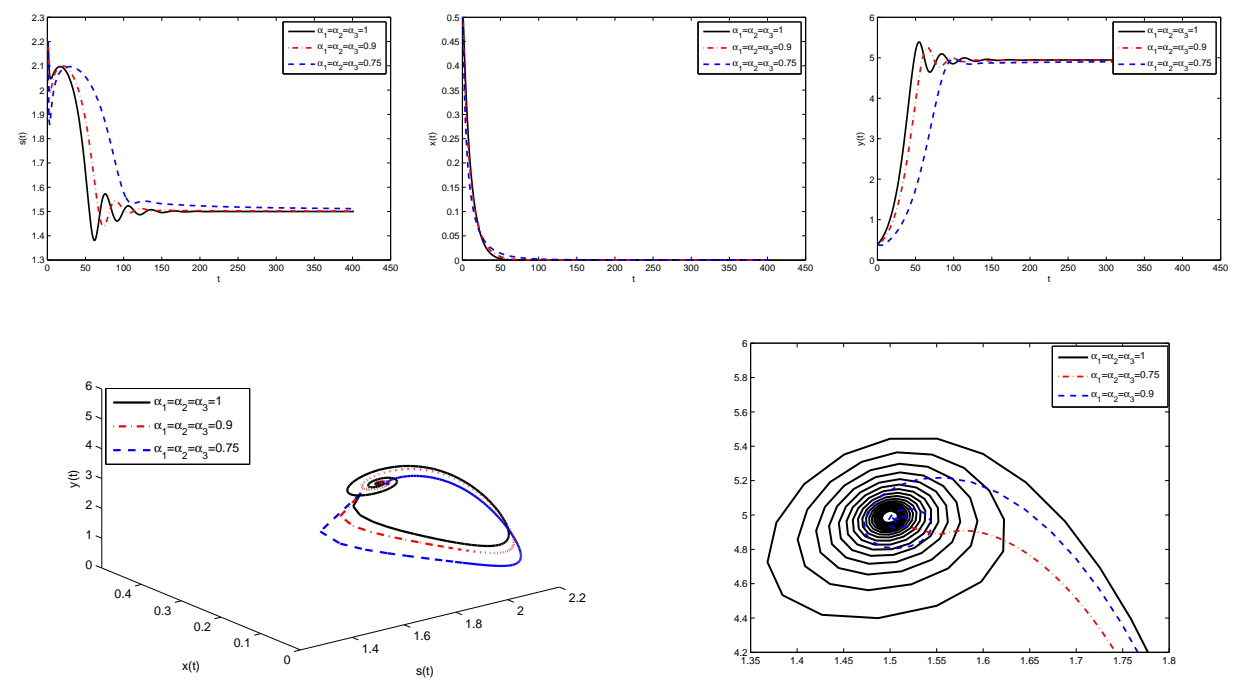

Figure 3. Numerical solutions of the system Eqs. (3.2) converge to the equilibrium point $E_{2}$ for different $\alpha_{i}$ with stepsize $h=1.5$.
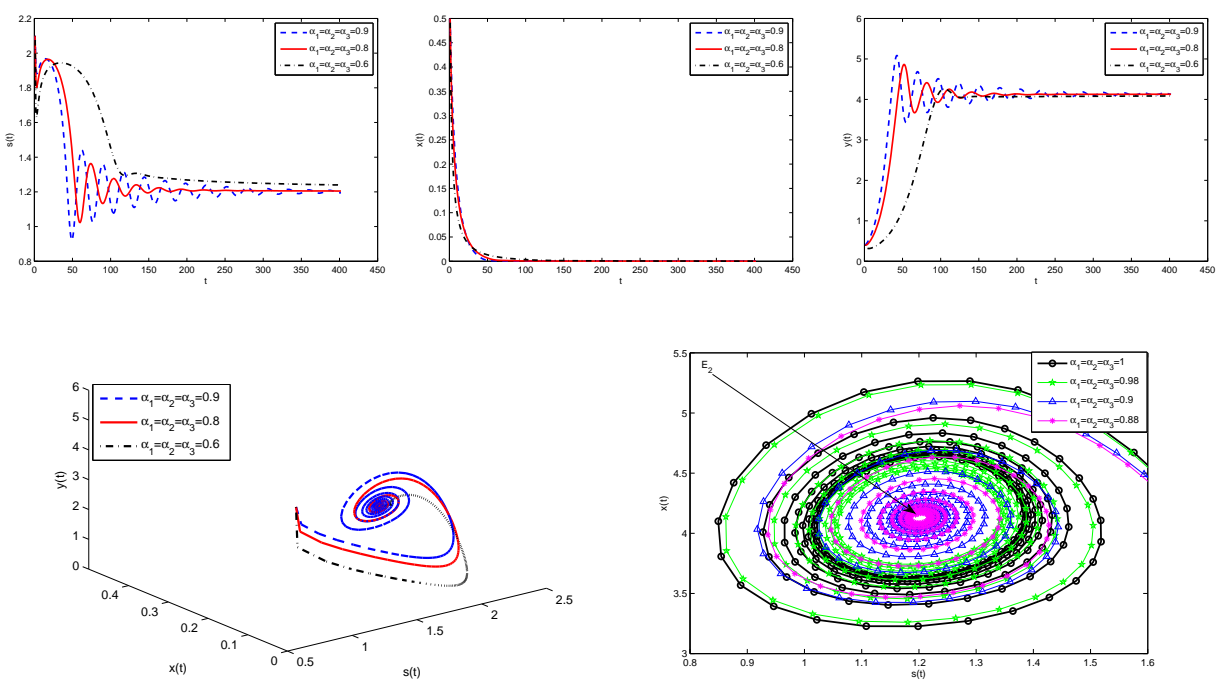

Figure 4. Numerical solutions of the system Eqs. (3.2) converge to the equilibrium point $E_{2}$ for different $\alpha_{i}$ with stepsize $h=1.5$ and $b_{3}=-0.274$

6.1. Remark. Note that the conditions $b_{2}, b_{3}>0$ are necessary for stability of the equilibrium points $E_{1}$ and $E_{2}$ when $\alpha_{i}=1$, but in obtained fractional-order model $b_{1}$ and $b_{2}$ might be less or equal to zero and the equilibrium points $E_{1}$ and $E_{2}$ are stable yet. We observe that for parameter values $A=0.0005, B=1.3, C=0.005, D=2$, 
$k_{1}=1, k_{2}=2.9, m_{1}=2, m_{2}=3, L=0.02, s_{0}=5$ and $Q=1.31$, the sing of $b_{2}$ is negative $\left(b_{2}=-0.0025\right)$, but for some $0<\alpha_{i}<1$ the numerical solutions of the system Eqs. (3.2) converge to the equilibrium point $E_{1}$. Also by using the values, $B=1.3$, $C=2, D=1.5, k_{1}=1, k_{2}=0.6, m_{1}=1.25, m_{2}=1.5, L=0.02, s_{0}=2.1$ and $Q=1$, the value of $b_{3}$ is less than zero $\left(b_{3}=-0.274\right)$, but the equilibrium point $E_{2}$ is stable yet (Proposition 3.2 and Figs. 4 and 5). Two dimensional plots $s-x$ illustrate better this matter.
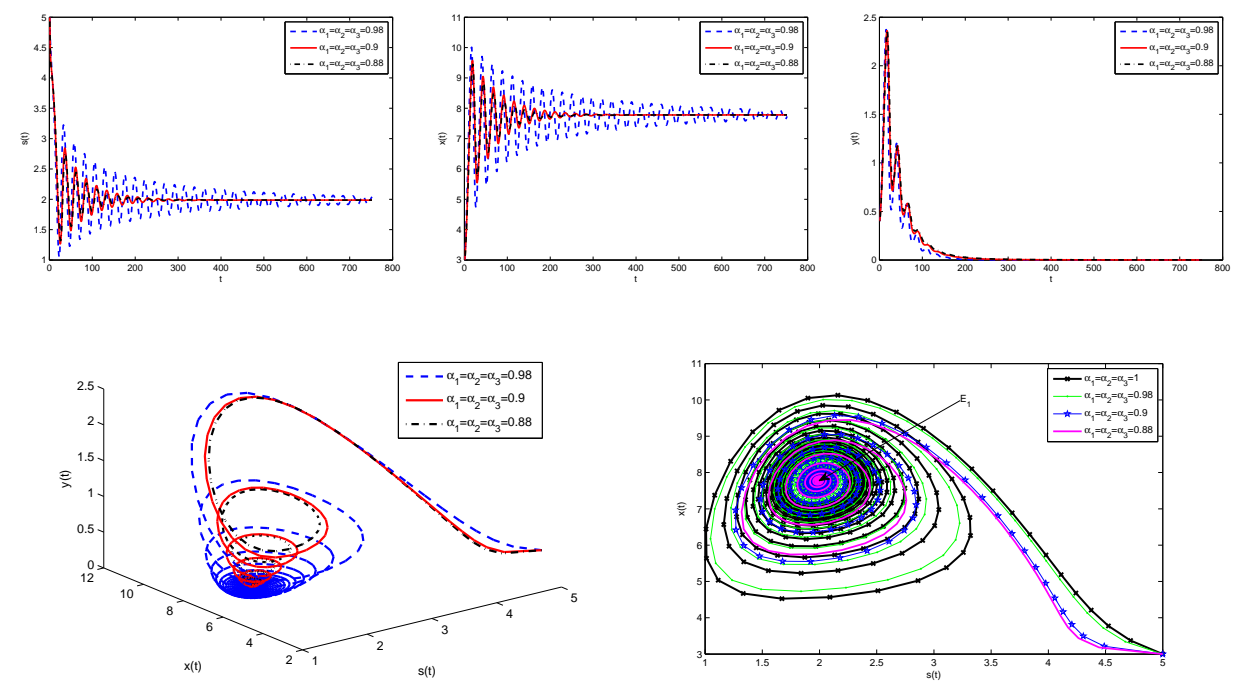

Figure 5. Numerical solutions of the system Eqs. (3.2) converge to the equilibrium point $E_{1}$ for different $\alpha_{i}$ with stepsize $h=0.8$ and $b_{2}=-0.0025$.

In Tabs. 1 3 the NSFD scheme for the system Eqs. (3.2) with forward Euler and fourth order Runge-Kutta methods are compared when $\alpha_{i}=1, i=1,2,3$. This comparison done for different time stepsize $h$ and the CPU times of the NSFD scheme are compared with forward Euler and fourth order Runge-Kutta schemes. As we observe, the CPU times of NSFD scheme are less than the CPU times of forward Euler and fourth order Runge-Kutta methods. Therefore the numerical solutions of NSFD scheme have better manner than Euler and Runge-Kutta methods. Also in Figs. 6 and 7, the forward Euler and fourth order Runge-Kutta methods are compared with NSFD scheme graphically. The stepsizes that used in these figures show the accuracy of the NSFD scheme.

\section{Conclusion}

In this paper the fractional form of the chemostat model is introduced. The stability of the equilibrium points is studied. Exploiting the NSFD scheme, we study the stability analysis as well as the dynamic behaviour of mentioned system. Moreover the obtained numerical results of NSFD scheme are compared with forward Euler and fourth order Runge-Kutta methods in integer-order case. Numerical results show the accuracy and efficiency of the NSFD scheme. 

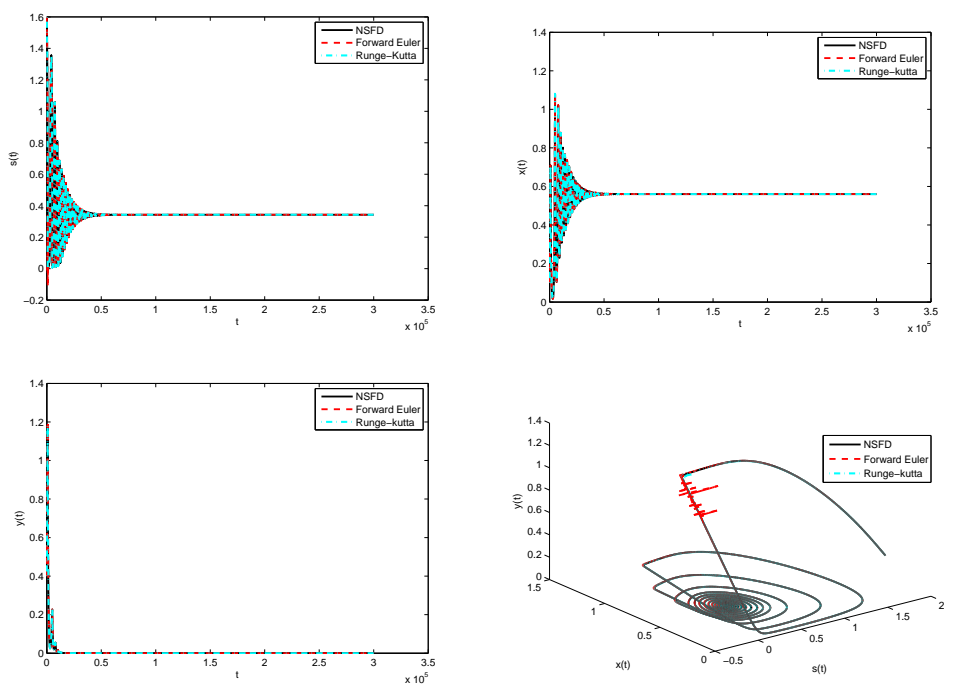

Figure 6. Numerical solutions with step size $h=0.002$ for forward Euler and fourth order Runge-Kutta and step size $h=0.005$ for NSFD methods converge to the equilibrium point $E_{1}$.
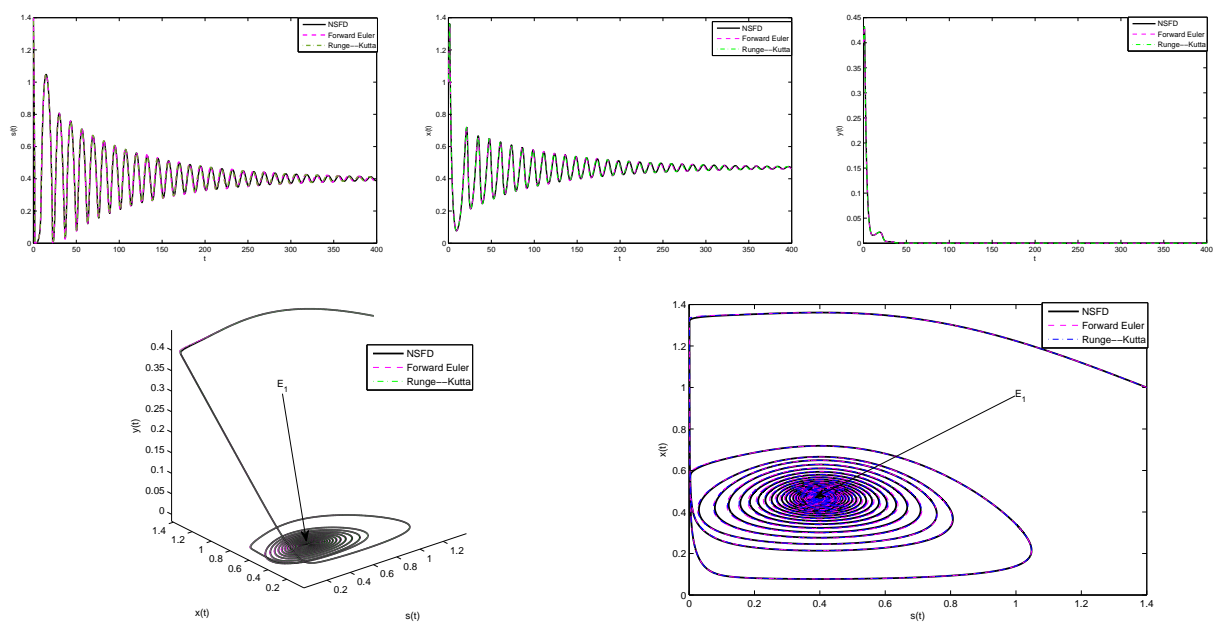

Figure 7. Numerical solutions with step size $h=0.002$ for forward Euler and fourth order Runge-Kutta and step size $h=0.02$ for NSFD methods converge to the equilibrium point $E_{1}$. 


\begin{tabular}{ccccccc}
$\mathrm{h}$ & Euler & CPU time & Runge-Kutta & CPU time & NSFD & CPU time \\
\hline 0.5 & Convergence & 0.004342 & Convergence & 0.005209 & Convergence & 0.000186 \\
1 & Convergence & 0.001203 & Convergence & 0.003202 & Convergence & 0.000094 \\
2 & Divergence & & Convergence & 0.001018 & Convergence & 0.000053 \\
2.5 & Divergence & & Convergence & 0.001018 & Convergence & 0.000053 \\
5 & Divergence & & Divergence & & Convergence & 0.000008 \\
10 & Divergence & & Divergence & & Convergence & 0.000003
\end{tabular}

Table 1. Qualitative result of the equilibrium point $E_{0}$ for different stepsizes $h$.

\begin{tabular}{ccccccc}
$\mathrm{h}$ & Euler & CPU time & Runge-Kutta & CPU time & NSFD & CPU time \\
\hline 0.001 & Convergence & 0.016342 & Convergence & 0.032029 & Convergence & 0.002066 \\
0.002 & Convergence & 0.015242 & Convergence & 0.022028 & Convergence & 0.002024 \\
0.025 & Convergence & 0.013242 & Convergence & 0.021011 & Convergence & 0.002003 \\
0.003 & Divergence & & Divergence & & Convergence & 0.002002 \\
0.5 & Divergence & & Divergence & & Convergence & 0.001103 \\
5 & Divergence & & Divergence & & Convergence & 0.000023 \\
10 & Divergence & & Divergence & & Convergence & 0.000023
\end{tabular}

Table 2. Qualitative result of the equilibrium point $E_{1}$ for different stepsizes $h$.

\begin{tabular}{ccccccc}
$\mathrm{h}$ & Euler & CPU time & Runge-Kutta & CPU time & NSFD & CPU time \\
\hline 0.01 & Convergence & 0.003423 & Convergence & 0.008292 & Convergence & 0.001146 \\
0.02 & Divergence & & Divergence & & Convergence & 0.000956 \\
0.5 & Divergence & & Divergence & & Convergence & 0.000123 \\
5 & Divergence & & Divergence & & Convergence & 0.000054 \\
10 & Divergence & & Divergence & & Convergence & 0.000004 \\
50 & Divergence & & Divergence & & Convergence & 0.000003
\end{tabular}

Table 3. Qualitative result of the equilibrium point $E_{2}$ for different stepsizes $h$.

\section{References}

[1] E. Ahmed, A. M. A. El-Sayed and H. A. A. El-Saka, On some Routh-Hurwitz conditions for fractional order differential equations and their applications in Lorenz, Rössler, Chua and Chen systems, Physics Letters A, 358 (2006), 1-4.

[2] E. Ahmed, A. Hashish and F. A. Rihan, On fractional order cancer model, Journal of Fractional Calculus and Applied Analysis, 3 ( 2012), 1-6.

[3] A. A. M. Arafa, S. Z. Rida and M. Khalil, Fractional modelling dynamics of HIV and CD4+ T-cells during primary infection, Nonlinear Biomedical Physics,6 (2012), article 1.

[4] J. Arino, S. S. pilyugin and G. S. K. Wolkowicz, Considerations on yield, nutrient uptake, cellular growth and competition in chemostat models, Can. Appl. Math. Quart. 11 (2003), $107-142$.

[5] K. Assaleh and W. M. Ahmad, Modeling of speech signals using fractional calculus, in: Proceedings of the 9th International Symposiumon Signal Processing and its Applications (ISSPA'07), Sharjah, United Arab Emirates, February 2007. 
[6] K. S. Cole, Electric conductance of biological systems, Cold Spring Harbor Symposia on Quantitative Biology, 107-116, 1993.

[7] W.-C. Chen, Nonlinear dynamics and chaos in a fractional-order financial system, Chaos, Solitons and Fractals, 36 ( 2008), 1305-1314.

[8] A. M. A. El-Sayed,A. E. M. El-Mesiry, and H. A. A. El-Saka, On the fractional-order logistic equation, Applied Mathematics Letters, 20 ( 2007), 817-823.

[9] H. Xu, Analytical approximations for a population growth model with fractional order, Communications in Nonlinear Science and Numerical Simulation, 14 (2009), 1978-1983.

[10] L. Debnath, Recent applications of fractional calculus to science and engineering, International Journal of Mathematics and Mathematical Sciences, 54 ( 2003) 3413-3442.

[11] R. Hilfer, Applications of Fractional Calculus in Physics, World Scientific, Singapore, 2000.

[12] Y. Ferdi, Some applications of fractional order calculus to design digital filters for biomedical signal processing, Journal of Mechanics in Medicine and Biology, 12 ( 2012), Article ID 12400088, 13 pages.

[13] X. Huang and L. M. Zhu, Bifurication in the stable manifold of the bioreactor with nth and mth order polynomial yields, Journal of Mathematical Chemistry, 46 (2009), 199-213.

[14] W. Lin, Global existence theory and chaos control of fractional differential equations, Journal of Mathematical Analysis and Applications, 332 ( 2007), 709-726.

[15] D. Matignon, Stability result on fractional differential equations with applications to control processing, Computational engineering in systems applications, 1996, 963-968.

[16] R. E. Mickens, Calculation of denominator functions for nonstandard finite difference schemes for differential equations satisfying a positivity condition, Numerical Methods for Partial Differential Equations, 23 (2007), 672-691.

[17] R. E. Mickens, Discretizations of nonlinear differential equations using explicit nonstandard methods, Journal of Computational and Applied Mathematics, 110 (1999), 181-185.

[18] R. E. Mickens, Advances in the Applications of Nonstandard Finite Difference Schemes, Wiley-Interscience, Singapore 2005.

[19] R. E. Mickens, Applications of Nonstandard Finite Difference Schemes, Singapore 2000.

[20] R. E. Mickens, A. Smith, Finite-difference models of ordinary differential equations : influence of denominator functions, J. Franklin Inst., 327 (1990), 143-149.

[21] R. E. Mickens, Nonstandard Finite Difference Models of Differential Equations World Scientific, 1994

[22] S. S. Pilyugin and P. Waltman, Multiple limit cycles in the chemostat with variable yield, Math. Biosci., 182 (2003), 151-166.

[23] I. Podlubny, Fractional Differential Equations, Academic Press, New York 1999. .

[24] H. Sheng, Y. Q. Chen and T. S. Qiu, Fractional Processes and Fractional-Order Signal Processing, Springer, New York, NY, USA, 2012.

[25] H. L. Smith and P. Waltman, The Theory of the Chemostat, Cambridge Univ. Press, Cambridge 1995.

[26] S. B. Yuste, L. Acedo and K. Lindenberg, Reaction front in an $A+B \longrightarrow C$ reactionsubdiffusion process, Physical Review E, 69 (2004), Article ID 036126. 